\title{
Capacitance of Atomic Junctions
}

\author{
Jian Wang, ${ }^{1}$ Hong Guo, ${ }^{2}$ Jose-Luis Mozos, ${ }^{2,3}$ C. C. Wan, ${ }^{2}$ Gianni Taraschi, ${ }^{2}$ and Qingrong Zheng ${ }^{1}$ \\ ${ }^{1}$ Department of Physics, The University of Hong Kong, Pokfulam Road, Hong Kong, China \\ ${ }^{2}$ Center for the Physics of Materials and Department of Physics, McGill University, Montreal, Quebec, Canada H3A 2T8 \\ ${ }^{3}$ Laboratory of Physics, Helsinki University of Technology, FIN-02150 Espoo, Finland
}

(Received 12 November 1997)

\begin{abstract}
We report the behavior of the electrochemical capacitance for a variety of atomic junctions using $a b$ initio methods. The capacitance can be classified according to the nature of conductance and shows a remarkable crossover from a quantum dominated regime to that of a classical-like geometric behavior. Clear anomalies arise due to a finite density of states of the atomic junction as well as the role played by the atomic valence orbitals. The results suggest several experiments to study contributions due to quantum effects and the atomic degree of freedom. [S0031-9007(98)06031-1]
\end{abstract}

PACS numbers: 73.40.Cg, 61.16.Ch, 61.43.Bn

For a very small conductor in which quantum effects play a role, it is not difficult to imagine that capacitance of the conductor may behave differently from the familiar classical case. For a small conductor its discrete nature of electron energy levels can be important, and the quantum correction due to the finite density of states (DOS) of the plates is known as the quantum capacitance [1,2]. For a microscopic sized conductor in which quantum coherence is maintained, one must consider the role played by the leads which connect the conductor to the outside world. One also needs to consider the finite screening length of the interacting electrons when it is not small compared with the system size. At these very small length scales, the relevant capacitance is the electrochemical capacitance $C \equiv$ $e d Q / d \mu$, which is a quantity depending on the electronic properties of the conductor [2].

Capacitance plays a central role in many phenomena such as the Coulomb blockade, and it is very important for several experimental techniques. However, there have been no quantitative predictions of capacitance for microscopic systems. Thus, we do not yet know its dependence on the atomic valence orbitals, the environment (e.g., the leads), and the shape, size, and other properties of a nanosystem. The purpose of this work is to make this connection by theoretically investigating capacitance of atomic junctions. In particular we shall study junctions formed by a small cluster of atoms, shown in the right panel of Fig. 1, where the clusters are sandwiched in between two metallic leads. We emphasize small system size where quantum effects are dominant and we answer a number of very relevant questions: (i) What is the value of the capacitance of these atomic junctions? (ii) What is the effect of atomic orbitals? (iii) How do we characterize the behavior of the capacitance? (iv) For the tip-substrate system familiar to scanning tunneling microscopy (STM), what is the $C=C(d)$ ? Although atomic nanosystems are of great current interest [3], these fundamental questions, which are important to ac transport, have not been addressed. It is clear that capacitance and many other mechanical and electrical properties of atomic junctions can be obtained only from detailed first principle analysis [4]. This will be our approach.

The theoretical analysis consists of four steps. First, we determine the atomic cluster shape by extensive quantum molecular dynamics (QMD) simulation using ab initio methods [5] with the usual local density approximation and pseudopotential [6]. The QMD found the global energy minimum such as the 3-atom triangle and 4-atom square (Fig. 1), as well as many local minima such as the other junctions. Second, we determine the scattering potential $V_{\text {eff }}(\mathbf{r})$ which is the mean field for all the electrons including those inside the leads [7]. Third, using $V_{\text {eff }}$ which defines the atomic junction plus the leads, we solve the single electron scattering problem [7]. The solution gives the scattering wave function as well as the scattering probabilities which, by Landauer formula, computes the conductance. Fourth, to obtain the electrochemical capacitance, we determine the nonequilibrium charge distribution $d Q$ inside the system due to an external perturbation on the chemical potential $d \mu$. $d \mu$ has two effects [2]: it can inject an amount of charge into the scattering junction, and such an injection produces an induced charge. While the net total charge is zero due to charge conservation, there could be charge polarization established which is referred to as the nonequilibrium charge distribution [8]. $d Q$ is found by solving [2] a 3D self-consistent Poisson equation for the characteristic potential $u_{j}(\mathbf{r})$, which describes the variation of the scattering potential landscape due to a change of the electrochemical potential at lead $j$. We solved it using a multigrid method. Finally, the electrochemical capacitance is obtained by the following formula [9]:

$$
C=\int_{\Omega_{1}}\left[\frac{d n_{1}(\mathbf{r})}{d E}-\frac{d n(\mathbf{r})}{d E} u_{1}(\mathbf{r})\right] d \mathbf{r},
$$

where the right hand side is just the nonequilibrium charge $d Q$ divided by $d \mu_{1}$, and the integration volume $\Omega_{1}$ is restricted to regions where $d Q$ is positive (or negative). The quantity $d n_{j} / d E$ is the partial local DOS and $d n / d E$ is the total local DOS. They are determined by the scattering 


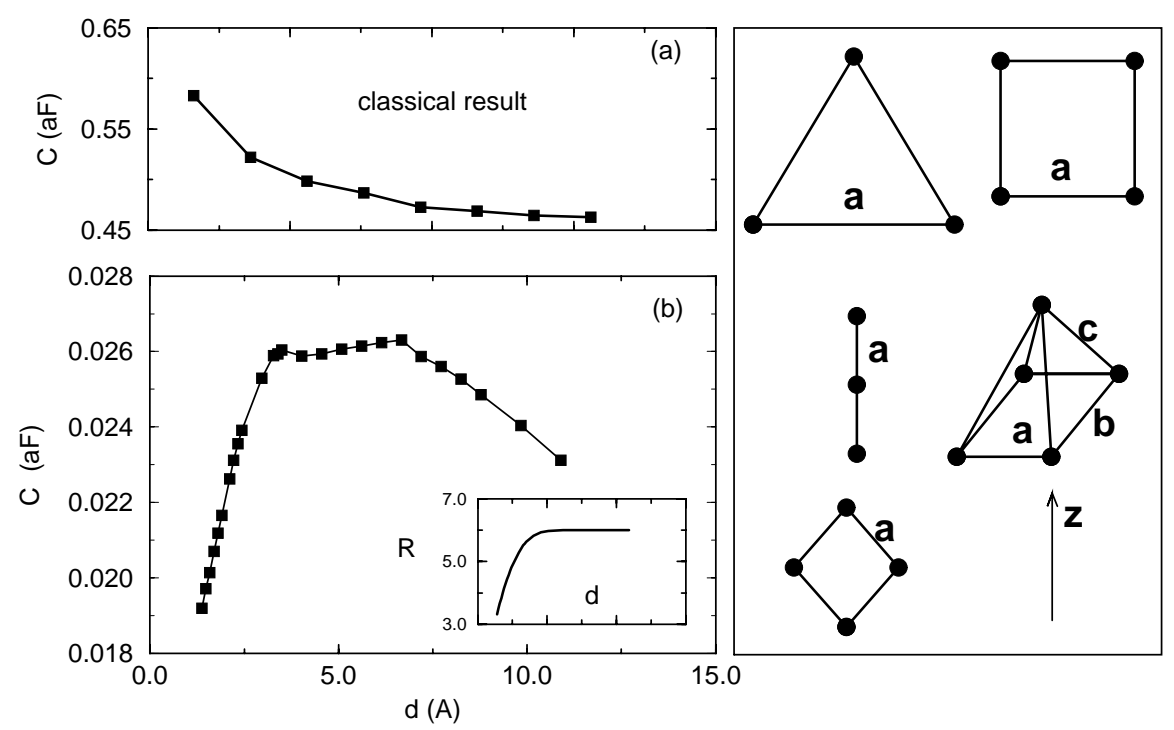

FIG. 1. Left panel: The electrochemical capacitance $C$ for the 5-atom tip-substrate system as a function of the distance $d$ between the tip and the substrate; (a) classical result; (b) quantum result. Inset to (b): The total reflection coefficient $R$ as a function of $d$. Right panel: The Al atomic clusters obtained from QMD simulations. They are sandwiched in between two metallic electrodes along the $z$ direction to form atomic junctions. For 3-atom triangle, $a=2.51 \AA$; for 3-atom chain, $a=2.75 \AA$; for 4 -atom rhombus, $a=2.54 \AA$ and the smaller angle is $66.3^{\circ}$; for 4 -atom square, $a=2.65 \AA$; for 5-atom "tip," $a=2.58 \AA, b=2.75 \AA$, $c=2.65 \AA$.

wave functions. Finally, for the special case when there is only capacitive coupling between the $+d Q$ and $-d Q$ regions, Eq. (1) reduces to the formula derived in Ref. [2].

We have examined six atomic junctions: five of them are $\mathrm{Al}$ junctions shown in Fig. 1 while the sixth is a single $\mathrm{Si}$ atom junction. The leads are simulated using the jellium model [7]. The cross section of a lead is $5.1 \times$ $5.1 \AA^{2}$, length $L=12.46 \AA$ for $\mathrm{Al}$ and $16.28 \AA$ for $\mathrm{Si}$. The jellium charge density is specified by the usual parameter $r_{s} \approx 1.1 \AA$, mimicking high density electrodes. The whole system is put into a supercell of size $11.76 \times$ $11.76 \times(2 L+2 d+a) \AA^{3}$ for standard plane-wave analysis to obtain the effective potential $V_{\text {eff }}$. Here $a$ is the length of the atomic clusters along the $z$ direction. For $\mathrm{Al}$ the lead-atom distance is fixed at the equilibrium value [10], $d=d_{o}=1.38 \AA$. For Si we used $d=3.65 \AA$ which is 3 times the equilibrium bond length [10]. Such a large $d$ creates two vacuum barriers surrounding the single $\mathrm{Si}$ atom. We thus obtain a single atom tunneling junction. With energy cutoff of 8 Ry we converged the total energy to better than $1 \mu \mathrm{eV}$. In the multigrid solution of $u_{1}(\mathbf{r})$, the finest grid consists of $65 \times 65 \times 257$ points, and the solutions are iterated to convergence with an error tolerance of $10^{-11}$.

Let us first consider the 5-atom tip junction and vary the tip distance $d$ to the other electrode. The classical result obtained by solving the electrostatic problem is shown in Fig. 1 which shows the expected monotonic decreasing behavior as $d$ is increased. Figure 1 also shows the quantum (nonclassical) result evaluated at the Fermi energy. Several observations are in order. First, at small $d, C(d)$ increases with $d$ which is clearly due to quantum effects: the tunneling from the tip to the substrate is reduced as $d$ increases. In other words, efficient tunneling at small $d$ diminishes the nonequilibrium charge distribution, hence reducing $C$. At intermediate $d$ when tunneling is essentially zero, $C(d)$ does not change very much with $d$, showing a compensating effect of the DOS and geometrical contributions to $C$. This could be due to the special arrangement of a tip shaped electrode. With even larger $d, C(d)$ reduces showing the behavior of geometrical-like capacitance. Figure 1 predicts a crossover of capacitance from quantum to classical-like regimes. Second, the relevant length scale is around $d \sim 5 \AA$, which is accessible using various surface probes. Third, the inset of Fig. 1(b) plots the total reflection coefficient $R$ (at $E_{F}$ ) as a function of $d$. Our results together with a semiclassical analysis [8], confirms $C(d) \sim R$ before the classical-like regime is reached. Finally, to experimentally observe the predicted nonclassical behavior, one must maintain both tunneling and small DOS. Assuming the semiclassical result [8] of the point contact, $C \sim R /\left[C_{o}^{-1}+D^{-1}\right]$, holds for our tunneling case, with $D=e^{2}(\mathrm{DOS})$ and $C_{o} \sim 1 / d$. Replacing $R$ with that appropriate for tunneling, $R \sim 1-\exp (-d / l)$, where $l$ is a system dependent parameter, we can roughly estimate the range of $d$ for which $C(d)$ increases with $d$. It is easy to confirm that when $D \ll C_{o}$ (atomic plates), we can have a reasonable and experimentally accessible range of order $l$. For the opposite $D \gg C_{o}$ case (macroscopic plates), nonclassical results show up only when $d<4 \times 10^{-3} l$.

Next we investigate the particularities due to the atomic degrees of freedom. We characterize the behavior of $C$ as a function of the scattering electron energy $E$ using 
the dc conductance $G(E)$ by fixing $d$. A reduction of $E$ corresponds to sampling less transport subbands, which is achieved during the elongation process of a typical nanowire experiment [3]. The solid and dotted curves of Figs. 2-4 show $C$ and $G$, respectively. The overall increase of $C$ as $E$ is increased is due to the fact that more transport channels enter the leads and possibly transporting through the atomic junction.

The six atomic junctions can be classified into three different transport categories. First, for the 4-atom rhombus and the single Si tunnel junction, $G(E)$ is dominated by quantum resonances. The behavior of $C(E)$ is sensitive to the same resonances (Fig. 2). For a single channel resonance at $E_{r} \approx-9 \mathrm{eV}, G=1 \times 2 e^{2} / h$. There, $C\left(E_{r}\right)=0$ (the upper right inset of Fig. 2). This is reasonable since if all the quantum wave can go through, the nonequilibrium charge cannot be established [8]. When there are more channels, e.g., at $E_{r} \approx-5.44 \mathrm{eV}$ for the $\mathrm{Al}$ case, and $-2.45 \mathrm{eV}$ for the $\mathrm{Si}$ case, $C\left(E_{r}\right)$ is reduced but not to zero. The resonance in the Al rhombus junction is due to the atomic point contacts (APC) at the two lead-atom connections. For the Si junction they are due to the $3 s$ and $3 p$ valence orbitals. The singlet $3 s$ gives a single conductance quanta, and the triplet $3 p$ is split by the electrodes into $3 p_{z}$ and $3 p_{x y}$, with resonances marked by $G\left(E_{3 p_{z}}\right) \approx 1$ and $G\left(E_{3 p_{x y}}\right) \approx 2$. This explains why capacitance has two nearby dips at $E_{r} \approx-2.45 \mathrm{eV}$ : these anomalies are due to atomic valence orbitals. For the 3-atom chain and 4-atom square junctions, the quasi-1D transport picture can be established where each perfect transmission channel gives a conductance plateau. The absence of the

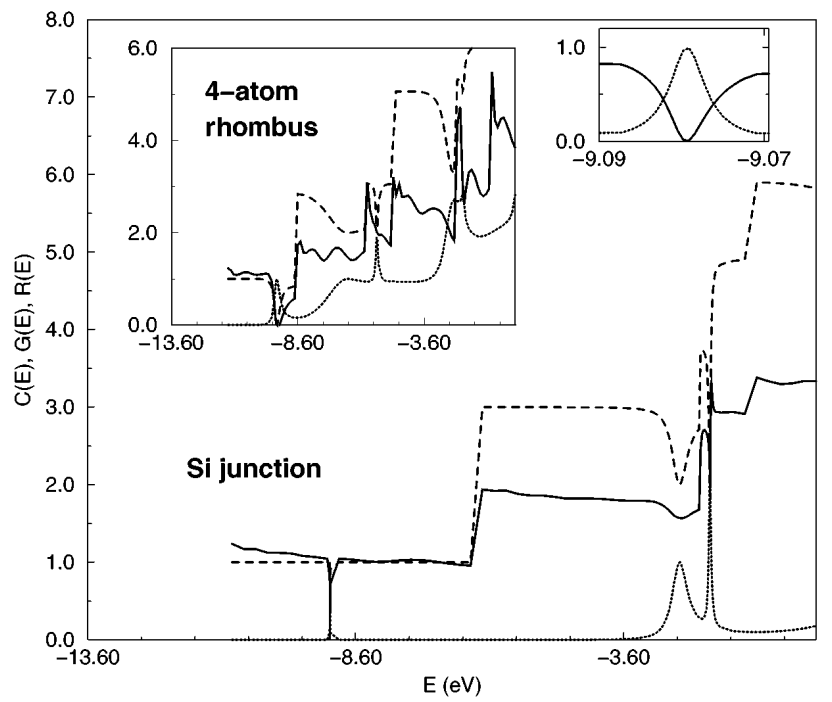

FIG. 2. Capacitances of the single $\mathrm{Si}$ tunneling junction and the 4-atom rhombus junction. These systems are characterized by resonance transmission. Solid lines: $C(E)$ in units of $0.01 \mathrm{aF}$. Dotted lines: $G(E)$ in units of $2 e^{2} / h$. Dashed lines: the total reflection probability $R$. The upper right inset is to resolve the extremely sharp $3 s$ resonance of the $\mathrm{Si}$ junction.
$G=2 \times 2 e^{2} / h$ plateau (Fig. 3) reflects the $D 4 h$ space symmetry of the 3-atom chain junction. $C$ also follows a rough "quantized" pattern if we average it over the energy range of a conductance plateau. For a plateau, there is a constant number of perfect quasi-1D channels; thus the local DOS inside the atomic junction does not change, leading to a rough steplike increase of $C$. Below we shall discuss the origin of fluctuations of $C$ on the plateau. For the 4-atom square junction the $D 4 h$ symmetry is broken, resulting in a more complicated transport subband structure in the atomic junction. Nevertheless the average capacitance also roughly follows a "plateau" increase. The third situation is provided by the 3 -atom triangle and 5-atom tip junctions. For them a quasi-1D transport picture cannot be established and there is no clear conductance quantization (Fig. 4). $G(E)$ increases almost monotonically except at a few energies where some resonances are caused by the APC formed by the tip atom and an electrode. In all categories it is clear that $C$ follows the behavior of the dc conductance $G$. Why does $C(E)$ have large fluctuations or jumps at certain energies? The reason is that $C$ is not only contributed by the atomic junction, but also by the electrodes in the quantum coherent regime. These special energies correspond to the transport subband edges of the electrodes where DOS changes discontinuously. For example, for the 3-atom chain there are three transport subbands inside the atomic junction (two of these are degenerate), but there are six subbands in the leads below the Fermi energy of the whole system. Thus, $G$ has two plateaus while $C$ shows more discontinuous changes as $E$ sweeps through each of the six subbands inside the leads. Hence, an interesting feature of the capacitance is its "discontinuous" behavior due to the opening up of more

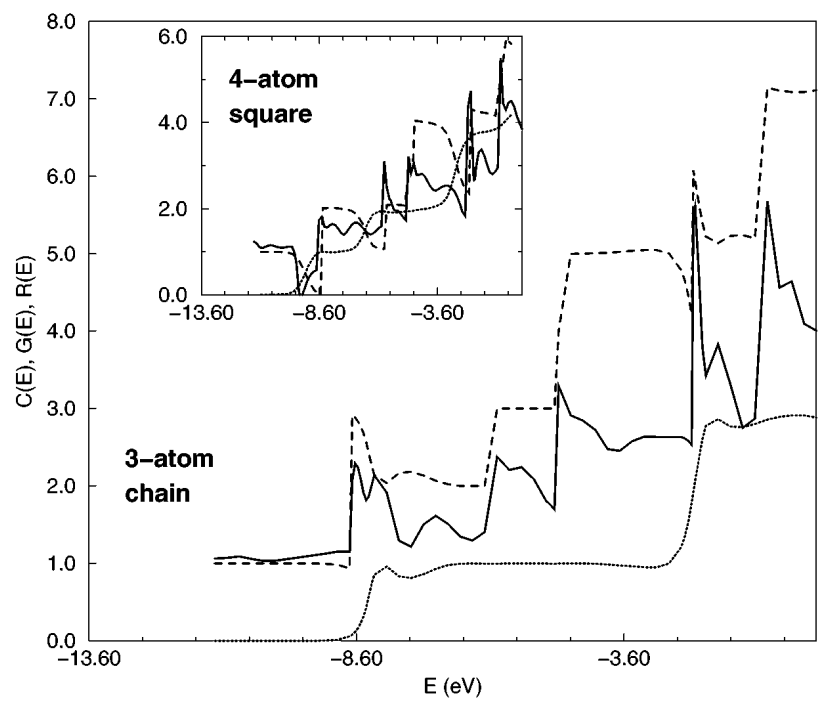

FIG. 3. $C$ (solid lines) of the 3-atom chain and the 4atom square junctions. For these systems quasi-1D transport channels can be established, as shown by $G$ (dotted lines). The inset is for the square junction. Dashed lines are $R$. Units are the same as those of Fig. 2. 


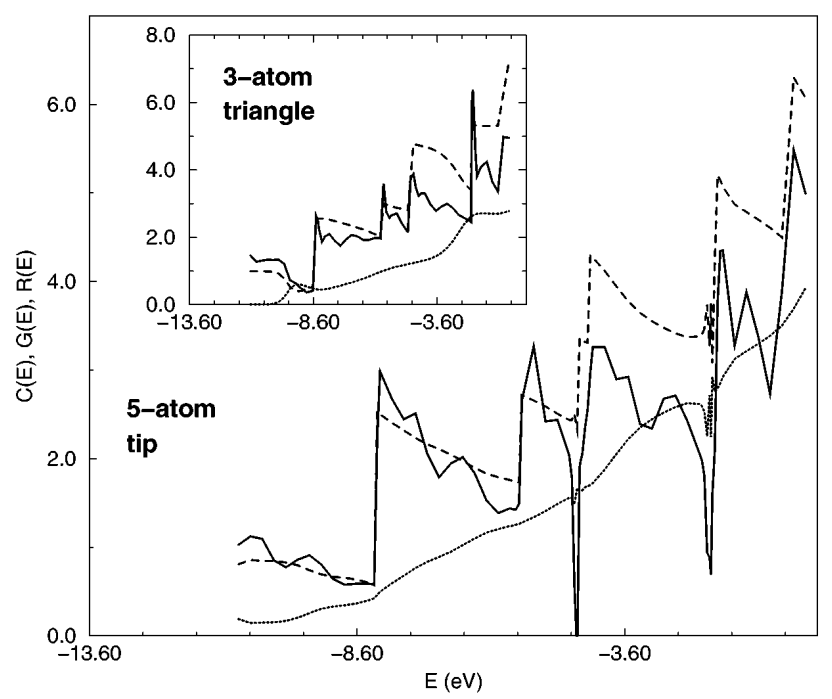

FIG. 4. $\quad C$ (solid lines) of the 3-atom triangle and 5-atom tip junctions. For them the quasi-1D transport channels cannot be established through the atomic junction, as shown by $G$ (dotted lines). The inset is for the 3-atom tip. Dashed lines are $R$. Units are the same as those of Fig. 2.

channels inside the leads although most of them cannot go through the atomic junction.

The atomic junctions have capacitance in the range of zero to $0.06 \mathrm{aF}$. However the classical geometrical capacitance $C_{o}$ can be several times larger than the total capacitance (Fig. 1). This difference is partly due to the finite DOS of our atomic scale plates. For two plates coupled purely capacitively (no dc current can go through), the geometrical and DOS contributions to capacitance are added in a series connection $[1,2]$; hence the total is less than individual parts. Our atomic capacitors allow dc transport, together with the very small DOS, resulting in much smaller values of $C$. In order to cross over to the classical values in addition to the classical behavior, much larger plates must be used for the capacitor such that the DOS is approaching the infinite limit as assumed by a classical theory.

To summarize, we have investigated the behavior of electrochemical capacitance for atomic sized junctions, using ab initio methods. Quantum corrections to capacitance originate from several factors: the small DOS resulting in a large screening length which establishes the nonequilibrium charge distribution, the direct DOS contribution to capacitance although our calculation predicts the total, the quantum coherence of the entire system which is the reason that leads also contribute to the behavior of $C$, and finally the role played by quantum tunneling effects. The functional dependence of $C=C(E)$ and $C_{E_{f}}(d)$ allows us to conclude that the classical notion of capacitance has been altered drastically at the atomic level. Our results further suggest several interesting experiments which should be accessible using STM and atomic-force microscopy techniques on atomic scale devices. First, the entire $C=C(d)$ curve should be measurable to demonstrate the remarkable crossover from quantum to classical-like behavior. If the tip and substrate can be made progressively larger, the crossover of capacitance values should also be accessible. Second, the discrete fluctuations of $C$ due to subband edges should be detectable in an elongation process on atomic wires using STM [3]. After an atomic wire is formed between a tip and a substrate [3], the subsequent elongation gradually suppresses transport subbands which is equivalent to decreasing the scattering electron energy of our calculation. The behavior of $C$ then gives direct information concerning the DOS of the atomic wire. Third, the anomalies due to atomic orbitals may be measurable in light of the single atom resistance experiment of Ref. [11]. These measurements should be able to correlate the behavior of $C$ to that of the conductance as predicted here. It is hoped that with these new experimental measurements one will be able to confirm the predictions here, and to obtain further information concerning the outstanding issues of quantum transport at the atomic scale.

This work was supported by NSERC of Canada, FCAR of Québec, the Croucher Foundation, and RGC of Hong Kong SAR under number HKU 7112/97P. H. G. thanks Professor R. Harris for useful discussions. We thank the Computer Center of University of Hong Kong for CPU allocations.

[1] F. Stern, IBM Research Report No. RC 3758, 1972; T.P. Smith et al., Phys. Rev. B 32, 2696 (1985); 34, 2995 (1986).

[2] M. Büttiker, J. Phys. 5, 9361 (1993).

[3] J. I. Pascual et al., Science 267, 1793 (1995); J. M. Krans et al., Nature (London) 375, 767 (1995).

[4] R. N. Barnett and Uzi Landman, Nature (London) 387, 788 (1997).

[5] O. F. Sankey and D. J. Niklewski, Phys. Rev. B 40, 3979 (1989).

[6] D. R. Hamman, M. Schll'uter, and C. Chiang, Phys. Rev. Lett. 43, 1494 (1979).

[7] C. C. Wan et al., Appl. Phys. Lett. 71, 419 (1997).

[8] T. Christen and M. Büttiker, Phys. Rev. Lett. 77, 143 (1996).

[9] This capacitance formula can be derived from the ac response theory presented in our recent work, Z.S. Ma, J. Wang, and H. Guo, cond-mat/9712060.

[10] N. D. Lang, Phys. Rev. B 52, 5335 (1995); N. D. Lang and A. R. Williams, Phys. Rev. B 18, 616 (1978).

[11] Ali Yazdani, D. M. Eigler, and N. D. Lang, Science 272, 1921 (1996). 